\title{
Research on the Teaching Methods of 3D Modeling Practice Skills
}

\author{
Cuan Ying \\ School of Computer Science, \\ Xi'an Shiyou University \\ Xi'an, China \\ e-mail: ying_cuan@xsyu.edu.cn
}

\author{
Song Wanting \\ School of Computer Science, \\ Xi'an Shiyou University \\ Xi'an, China \\ e-mail: 895227270@qq.com
}

\begin{abstract}
D Animation technology, as the major course of digital media technology, is technical and artistic. This paper takes 3d modeling and animation process of the smart watch as an example, combining the features of the smart watch (case, wristlet, buckle, hand, back, watchcase accessories .etc) with Chinese traditional culture, using 3D Max software and Vray renderer Technology. It realizes the $3 D$ animation design of smart watch, and has a certain guiding significance for the practical teaching of animation design course.
\end{abstract} skills

Keywords-Animation; 3DMax\&Vray; Design Plan; Practice

\section{INTRODUCTION}

3D Animation Technology is a course that studies how to use computer and professional software to design, produce and simulate the effect of $3 \mathrm{D}$ images. This course aims to introduce the main functions, operation methods and techniques about 3DS MAX software, including basic modeling, advanced modeling, edit modifiers, materials and maps, light and camera, motion controllers, particle systems, skinned mesh and special effects of video, etc. This course can help students work in the field of film production, advertising and multimedia production [1 3]. This course needs to focus on cultivating students' practical ability and artistic aesthetic ability, and strengthen practical training sessions in teaching. The course not only lets students understand the theoretical knowledge of animation art, but also highlights the practical training of design process and creative methods of $3 \mathrm{D}$ animation. This paper adopts $3 \mathrm{D}$ Max software and Vray renderer technology, and takes 3D animation design of smart watch as an example, through elaborating the modeling method and practice skill of 3D animation design of smart watch to make a guide effect of the practice teaching of animation design course [4 6].

\section{THE DESIGN OF SMART WATCH}

With the rapid development of modern high technology, especially the development of electronic technology and communication technology, microelectronic technology products have penetrated into the field of clocks and watches. At present, China is more and more important in the world. The design of the watch integrates Chinese elements and Chinese culture. Therefore, the design of a smart digital watch that contains elements of traditional Chinese culture highlights the Chinese characteristics [7 8]. It applies 3DS MAX to create 3D Model of the watch, so the structure, functions, features and working principles of the watch are presented to the user through the $3 \mathrm{~d}$ animation. Then customers can more intuitive understanding of traditional Chinese culture elements.

According to the characteristics of the smart watches, the basic appearance structure of the watch is divided into six items: case, wristlet, buckle, hand, back and watchcase accessories. In the design, Chinese traditional figure is used as the element of watch dial design and the Chinese ancient timing pattern used as timing of the watch. The chip of the watch is electronic integrated, so the watch cover is made of waterproof material, and the crystal uses anti-pressure glass with high strength .To meet the tastes of most people, this design does not use the traditional craft buckle strap, choose to adjust the opening of the strap eliminates the need for clasp production. 3DMAX is responsible for modeling the overall appearance and components of watches, as well as realizing the animation part of the intelligent function switch.

\section{MODELING AND ANIMATION MAKING}

\section{A. Design of Watch appearance}

The standard parts of the watch are commonly used parts. It includes case, crown, hand, buckle, wristlet, watchcase accessories and more. By extracting the structural features of the standard parts, the independent geometry parameters of each component could be determined. Under CAD environment a $3 \mathrm{~d}$ model can be established and the variations of independent dimension parameters can be set to implement parameter design of watch components. As is shown in Figure 1 , it described the standard size parameters of the 3D model [9 11].

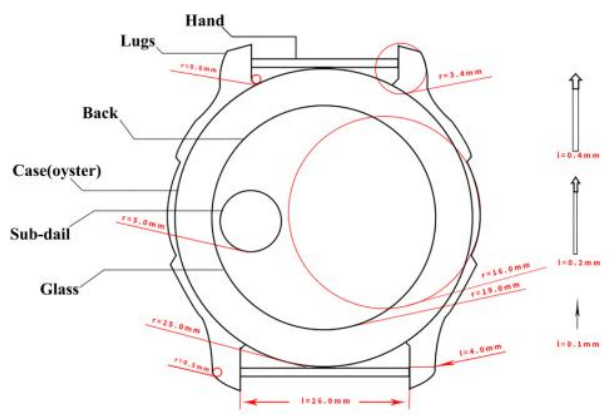

Figure 1. The standard structure design of watch 


\section{B. Modeling and materials}

In the standard structure design parameters of the watch, the basic structure of the watch is constructed by setting up the standard geometry of the cylinder in the 3D Max working scene. It uses the function of editing command such as shell, FFD and extrusion to accurate data size processing of the structure. Watch strap selection 2D modeling, because the 2D model can use Bezier to achieve precise control of the curvature of the value of the strap, and better turn into a $3 \mathrm{D}$ model. For a better abutment of all the watch parts, tools such as level and fast abutment are used in the processing.
Because the watches each part contains the glass, the metal, the aluminum alloy, the plastic steel and so on many kinds of material. Using the high quality rendering software produced by Chaos group and As gvis company, the real material simulation of the watch can be realized by controlling the properties of different material parameters. [12 14]. Due to the different physical properties of each part of the watch, the reflection and refractive index of the light are different, so the special material VrayMtl material provided by the Vray renderer is used, and different physical lighting parameters are needed for different materials in the 3D Max environment. The structure and material parameters of watch components are shown in Table 1.

TABLE I. WATCH COMPONENT STRUCTURE MATERIAL PARAMETERS

\begin{tabular}{|c|c|c|c|c|c|c|}
\hline & Component structure & Material & Reflectivity & Glossiness & $\begin{array}{c}\text { Index of } \\
\text { refraction }\end{array}$ & Remarks \\
\hline \multirow{5}{*}{ Exterior parts } & Case & $\begin{array}{c}\text { Stainless steel } \\
\text { (high reflective) }\end{array}$ & 150 & 1.00 & $\mathrm{X}$ & \\
\hline & Crown & Aluminium alloy & 85 & 0.75 & $X$ & \\
\hline & Hand & Stainless steel & 150 & 1.00 & $\mathrm{X}$ & \\
\hline & Back & Ceramic & 133 & 0.95 & $\mathrm{X}$ & $\begin{array}{c}\text { Fresnel } \\
\text { phenomenon }\end{array}$ \\
\hline & Buckle & $\mathrm{X}$ & $\mathrm{X}$ & $X$ & $\mathrm{X}$ & $\mathrm{X}$ \\
\hline \multirow{3}{*}{$\begin{array}{l}\text { Watchcase } \\
\text { accessories }\end{array}$} & Glass & Perspex & 120 & 0.95 & 252 & Fall off \\
\hline & Wristlet & Rubber & 30 & 0.6 & $\mathrm{X}$ & Fall off \\
\hline & Bezel & Resin & & 0.95 & & Fall off \\
\hline
\end{tabular}

\section{Implementation effect}

3D Max \&Vray technology is used in Smart watches overall appearance, structure modeling and intelligent switching functions, it brings the watch making technology to the screen and shows to consumers vividly and rich of artist, Consumers can understand the dynamic characteristics and functions of watches intuitively, comprehensively and comprehensively. It allows customers to feel the novelty, goodwill and trust, so that the audience in a short time to understand the advantages of the product. It also establishes a brand image and leads to a better dissemination of the of Chinese cultures and traditional elements.

The smart watch uses 3D Max to make animation time axis, key frame and camera tracking animation. It makes the whole appearance of the watch, the display of each component structure and the switch of watch function. The effects of the rendered watch are shown in Figure 2.

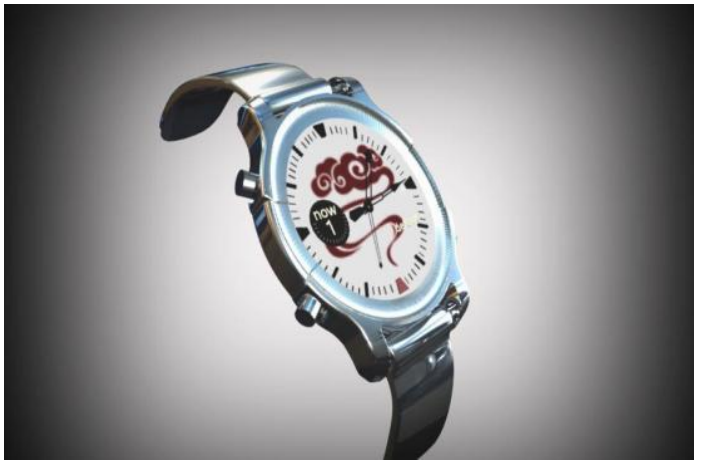

Figure 2. Render the processed watch effect

\section{ANALYSIS OF TEACHING PRACTICE}

The main purpose of 3D Animation Technology course is to enable students to accomplish the process of animation project independently through practical training, and cultivate their practical ability and creative thinking. It has a higher requirements for students' practical ability and innovative ability. Through classroom teaching and practice projects closely linked, first explain the role of the scene design ideas, and then explain the modeling, from easy to hard. The smart watch design focus on the structural level analysis, the model from simple to complex, just as the accumulation of building blocks the same. The practical training emphasizes the standardization of the size and specifications, the apparent absorbs the design of Chinese traditional elements, and the material model design is 
strengthened. Some individual difficult designs should be appropriate decomposition or be focused on, then the integration of model design will be finished gradually, this called practical training projects drive teaching.

\section{CONCLUSION}

In this paper, the $3 \mathrm{~d}$ animation design of smart watch is used as an example, and the $3 \mathrm{~d}$ animation design technique of smart watches is described in detail. The design fully displays the characteristics of Chinese cultural elements. It realizes the course teaching by practice, integrates teaching with learning, raises students' learning interest and practical ability, and has certain guiding significance for the practical teaching of animation design course.

\section{REFERENCES}

[1] Liu Xiao-qian, Cao Wei-quan, Zhang-Lu. The Modeling and Animation of Complex 3D Forestry Scenarios[J]. Journal of Image \& Graphics, 2010(1)

[2] Lake A, Marshall C, Harris M, et al. Stylized rendering techniques for scalable real-time 3D animation[C]// 2000:13-20.

[3] Laga H, Tabia H. Modeling and Exploring Co - variations in the Geometry and Configuration of Man - made 3D Shape Families[J]. Computer Graphics Forum, 2017, 36(5):13-25.

[4] Liu Xian-mei, Huang Jing, Liu Xiao-ming. Discussing of Differences Between 3-D Animated Technology and 3-D Virtual Technology[J]. Computer Simulation, 2004, 21(9):127-130
[5] Guo Xue-kun, Chen Hao, Qiu Tian, et al. Journal of Computer-Aided Design \& Computer Graphics[J]. Journal of Computer-Aided Design \& Computer Graphics, 2017, 29(7):1177-1185.

[6] $\mathrm{Xu} \mathrm{C}$, Lyons K. Shimmering Smartwatches: Exploring the Smartwatch Design Space[C]// International Conference on Tangible. ACM, 2015:69-76.

[7] Shi Y. Cultural Elements in Chinese and English Metaphors[J]. Jounal of Ningbo University, 2000.

[8] Iskin I, Dahlawi Y, Fenwick D, et al. Exploring the influence of cultural elements on Chinese manufacturing quality[J]. Journal of Technology Management in China, 2010, 5(2):141-157.

[9] Wang Wei-wei, Wang Yi-ru, Yang Xiao-yan. Study on the Application of Tang Mirror Culture in the Design of Wrist Watch[J]. Beauty \& Times, 2015(10):100-102.

[10] Song L I, Yang Z, Wei Y M, et al. Survey of sketch-based technique aiding 3D animation[J]. Application Research of Computers, 2010, 27(9):3210-3214

[11] Zhou Wen-xuan, Xia Jing, Wang Qiang. Children's smart watch features and appearance of the program design[J]. Technology Innovation and Application, 2017(15):18-18.

[12] Hu Xi-wei. Theory Research of Three dimensional Cartoon and Virtual-reality Technology[D]. Wuhan University, 2005.

[13] Chen Hong-hong, Guo Hong-fei. About Three-dimensional Simulation of Materials Research and Discussion[J]. Computer Knowledge and Technology, 2011, 07(7):1632-1634.

[14] $\mathrm{Hu}$ Meng-zheng, Song Jing-yu. Research and Implementation of Virtual Reality Modeing Technology Based on 3ds Max[J]. Industrial Control Computer, 2011(6):114-116. 